\title{
On the Practice and Exploration of the Management Mode of Compact Medical Association
}

\author{
Jing Jing \\ The Second Affiliated Hospital of Shaanxi University of Traditional Chinese Medicine, Xianyang, Shaanxi \\ Province, 712000
}

Keywords: Public Hospital; Compact Medical Association; Practice and Exploration

\begin{abstract}
At present, there are various flourishing models to develop the medical associations at different places, and each has its merits and deficiencies. As a third-grade hospital of integrated traditional Chinese and Western medicine, the Second Affiliated Hospital of Shaanxi University of Traditional Chinese Medicine is concerned with the responsibility of people's livelihood and the health and well-being of the people, and it is also constantly exploring and innovating to make the medical association become an integration of services, responsibilities, interest and management. This paper intends to analyze and discuss the challenges, problems and strategies in the construction of close medical associations in hospitals.
\end{abstract}

In recent years, in response to the national grading diagnosis and treatment policy, promoting the specific implementation of quality resources, enhancing the interaction and cooperation with grassroots hospitals, our hospital actively constructs medical associations, and its function position is also constantly being adjusted and enriched after the construction, promotion and exploration stages.

\section{Current Status of Construction}

\subsection{Work Hard to Improve the Quality of Medical Services}

As a three-level hospital of Chinese and Western medicine, the third batch of national key integrated Chinese and Western medicine hospitals, the national standardized training base for Chinese medicine residents, the post-doctoral innovation base of Shaanxi Province, the demonstration unit of Shaanxi traditional Chinese medicine culture construction, and the second clinical practice of Shaanxi University of Traditional Chinese Medicine. The first-level discipline construction unit of medical school and clinical medicine consists of three parts: Xixian Branch, Qindu Branch and Xi'an Branch. Xixian Branch (Central Hospital of Xixian New District) covers an area of $300.96 \mathrm{mu}$, with 1,500 beds and a construction area of 230,000 $\mathrm{m}^{2}$. It is currently under construction and is scheduled to be put into use at the end of 2018. Qindu Branch has 656 beds and 696 beds, 45 clinical and medical departments, and 1211 employees, including 174 senior titles, 203 masters and 265 clinical teachers. The annual outpatient volume is 430,000. There were more than 34,000 person-times of patients discharged from the hospital. There are 1 regional TCM clinics (Tuina), 23 key disciplines and key specialties at all levels.

The hospital actively responds to the grading diagnosis and treatment strategy, making full use of the advantages of professionals in various disciplines of Shaanxi University of Traditional Chinese Medicine, and medical, teaching and scientific research go hand in hand. At the same time, we are striving to build a high-level integrated Chinese and Western medicine university affiliated hospital and regional medical treatment center with excellent comprehensive ability, outstanding combination of Chinese and Western medicine, outstanding characteristics, and first-class medical service, teaching, research, health management and cultural heritage. The service is to deepen the medical and health service system as the overall situation. With the goal of improving medical quality and service level, we will meet the needs of the masses for medical treatment, effectively promote the sinking of quality medical resources, and continuously improve the ability to connect 
and turn down, and strive to provide comprehensive and life-cycle health services for the people.

\subsection{Give Full Play to the Characteristics and Promote the Construction of Medical Groups}

In 2016, the hospital officially started the construction of the medical association. At the beginning of the year, the Shaanxi Second Affiliated Hospital Medical Group was established. There are 52 member units: one of the third-level hospitals, nine of the second-level hospitals, and 24 of the first-level hospitals. 13 service stations and 5 private hospitals. As the leader of Shaanxi Provincial Hospital of Integrated Traditional Chinese and Western Medicine and Xianyang City Maternity Hospital, the hospital actively played its special features and promoted the construction of specialist medical associations. Subsequently, the Xianyang City Obstetrics and Gynecology Association was established (33 members, including Level 3). There are 1 hospital, 26 secondary hospitals, 6 first-class hospitals, and Xianyang TCM Medical Union (currently 7 medical institutions). After the establishment of the medical group and the medical association, our hospital used technical support as a link, opened the official website, built a graded diagnosis and treatment platform, held a council meeting, formed a professional technical committee, incorporated high-level medical technicians, and established experts. We will help the system, carry out training for grassroots doctors, implement the graded diagnosis and treatment system, and strive to radiate quality resources.

\subsection{Take Various Measures to Improve the Connection Ability}

On March 5, 2017, Premier Li Keqiang pointed out in his government work report that "a comprehensive pilot of various forms of medical complex construction will be launched, and all three-level public hospitals should participate and play a leading role.” This marks the official rise of the medical association as a national policy. On April 23, 2017, the General Office of the State Council issued the "Guiding Opinions on Promoting the Construction and Development of Medical Complexes" (G.B.F. [2017] No. 32). On July 21 of the same year, the Office of the Shaanxi Provincial Government issued "Implementation Opinions of the General Office of the Shaanxi Provincial People's Government on Further Promoting the Construction and Development of Medical Complexes" (S.Z.B.F.[2017] No. 61). According to the requirements of the "Opinions", our hospital has responded with its own reality, actively responded, developed ideas and innovative measures, and reached an agreement with the Fengxi New Town Administrative Committee of the Xixian District Government. We must explore a new model of regional grading diagnosis and treatment according to local conditions, and strive to protect the health of the local people.

In December 2017, the hospital and the Fengxi New Town Management Committee jointly established the Medical Association of the New West of Fujian Province, taking the lead in piloting the innovation of the Xi'an model of the medical association. The organization is neither a loose medical association nor a close medical association, but a new type of medical association that is relatively close between the two, i.e., the Education and Health Bureau of the New West City Management Committee will assume the administrative powers of the member units, and entrust the medical services of the four public health centers of Dawang, Mawang, Gaoqiao and Diaoyutai in the jurisdiction to our hospital. In order to do this work, our hospital has transferred four business backbones to various health centers to serve as the vice president of the business, and is stationed in the hospital for daily business. A number of experts were selected to sit in the clinic, and the auxiliary hospitals completed a number of work such as signing a family doctor and building a Chinese medicine museum. As of September 2018, the number of experts sent by hospitals to various hospitals in Fengxi was 27 person/week. Through the "semi-hosting" Fengxi hospitals, our hospital and Fengxi New Town Management Committee officially started a close business connection, successfully decentralized manpower and technology to all parts of the jurisdiction, and established a reputation among the people in the jurisdiction.

\section{Existing Problems}

The hospital has continuously strengthened and promoted the construction of the medical 
association, and achieved certain results, but there are also many shortcomings. From the current medical operation of the medical association, there are four main problems:

\section{1 “Unreasonable” operation mechanism}

At present, nearly 100 members have signed a medical cooperation agreement with the hospital. The number of member units is large, the scope is large, and the organization is loose. Due to the lack of systematic medical management system and operational mechanism, there is a lack of strict medical management system coordination. When carrying out work, the hospital cannot deal with the management of members, people, finances and materials, and cannot carry out substantive medical cooperation and complete medical cooperation with all units.

\subsection{Unable to utilize advantageous resources}

At present, due to the imperfect system of various aspects such as specialist construction and personnel training among medical associations. In the work of promoting counterparts to help the grassroots level, it is difficult to mobilize the initiative and enthusiasm of the backbone of experts and the sinking of high-quality resources, and advantageous resources cannot be used for patients. In addition, due to insufficient support for the settlement policy of medical insurance, it also affects the utilization of superior resources.

\subsection{Basic hospitals cannot "handle"}

Due to institutional restrictions, the hospital of Fengxi New Town Medical Union only took over the medical business of the hospital. Due to the lack of performance and personnel, the hospitals gradually explored adaptation in the new and old modes and could not develop rapidly. The development of medical treatment technology is slow, the general qualifications of talents are low, and the level of medical services is not high. This cannot meet the needs of the referral, and all aspects require the support of the hospital, and the progress of the work is slow.

\subsection{Disconnection between "institutions"}

The limitation of objective conditions has made it difficult to implement. For example, the information sharing platform has not been realized, the information system is incompatible, and the medical information cannot be shared. The medical quality and inspection result standards are not uniform, and the inspection do not recognize each other, resulting in an increase in the medical cost of the patient; when the patient is transferred to the clinic, the visit information and records cannot be read from each other, and there is no interconnection between the residents' health information management. All of the above factors have affected the implementation of graded diagnosis and treatment.

\section{Thinking and Exploration}

Since last year, it has only been about a year or so, whether it is to promote the construction of urban medical associations, medical communities, specialist medical associations, or to strengthen telemedicine collaboration. The state is making great efforts to fundamentally solve the problem of people's medical difficulties. In the process of promoting the construction and development of a close-knit medical association, the author believes that the core is "linking", that is, through the management of the government, the construction of specialties, the cultivation of talents, the mechanism of interest, and the way of the Internet, the joint venture, joint effort and association are realized. And networking, and then build a new mechanism for high-quality resources up and down and left and right connectivity.

\subsection{Unity - an active “co-constricted” government}

As the central hospital of Xixian New District, the hospital will strengthen the cooperation between the government and the hospital, explore the construction of close medical associations according to local conditions, and strive to create a regional health service highland and create a 
new mode of medical joints. At present, the hospital has basically reached an agreement with the Xixian New District Management Committee, supported by the Xixian New District Administrative Committee, and the hospital takes the lead in coordinating medical institutions at all levels. Efforts have been made to break down barriers in management systems and medical insurance payments, improve and perfect the mechanism of cooperation among some parts of the medical association, and actively implement the link of talent sharing, technical support, mutual recognition, prescription flow, and service convergence. Change competition into synergy, strive to establish a network structure from top to bottom, and fully implement the grading diagnosis and treatment system, and promote the use of quality medical resources. On the basis of giving full play to the advantages of the characteristics, we will give full play to the social service functions of public hospitals, guide and take the lead in making the institutions and personnel within the medical associations become "families". Let the big family of the medical association actively provide unified, high-level and diversified medical services to the people in the region. At present, the hospital has used Fengxi New City as a pilot project for the construction of a new model of the Xixian New District of the Medical Union. It has achieved certain results in exploring the new mode of the Western Union.

\subsection{Joint Efforts - there is a mutual assisting “progressive” mechanism}

Starting from management, clinical, scientific research, teaching and other aspects, we will improve the top-level design and establish a system that is conducive to division of labor, resource sinking, service homogeneity, and continuous service. Actively carry out close cooperation and realize the sharing of resources such as technology and talents, so that the medical associations can truly "join the heart" and let the superior resources "have power, channel, and willing to go down". Let the primary health care institutions "provide technology, provide services, and be willing to pick up and accept", fundamentally improve the level of regional medical services to meet the needs of clinical and health services.

The first is to establish a system of establishment, homogenous management, establish and improve the rules and regulations of the entire medical association, clarify the responsibilities, authorities and obligations of all parties, strengthen institutional constraints, and promote the homogenization of regional medical services. The second is to integrate resources and innovation mechanisms at various levels such as discipline construction, personnel training, scientific research, and technological innovation. It has a flexible and rewarding salary system, a position setting for the upper and lower positions, a flexible and orderly flow of people, and a smooth and unobstructed transportation channel. The third is precision assistance and improvement. Through careful investigation, we will clarify the needs of member units to help them, and dispatch relevant experts to the grassroots level to carry out a series of work such as sitting, warding, teaching, and surgery, and explore the establishment of an effective business guidance and cooperation model. This can effectively enhance the service capacity and level of primary health care institutions.

\subsection{Share Benefits — develop a "win-win" vision}

According to the spirit of the "Notice on Printing and Distributing the Comprehensive Performance Appraisal Work Plan (Trial) of the Medical Complex" (National Health Medical News [2018] No. 28) issued by the government, the hospital will use economic leverage to use assessment criteria and indicators as the main line of daily work. Through the institutional mechanism innovation and the adjustment of the interest structure, everyone has the willingness to voluntarily, voluntarily, and find ways to help each other and truly establish a medical complex with stable operation, risk sharing, benefit sharing and sustainable development. This can really achieve a gratifying situation in which patients are referred to the order.

\subsection{Networking - there is a national "sharing" exploration}

The hospital made great efforts to invest nearly 70 million special funds for the construction of smart hospitals. While improving the information support system, we will coordinate the interconnection of information systems between medical institutions within the medical association and actively explore Internet medical care. Strive to effectively support collaborative applications 
and services such as appointments and treatments, two-way referrals, chronic disease management, and fund settlement within the medical association. At the same time, the hospital plans to establish a third-party imaging center, inspection center, remote consultation center, family doctor contract service platform, patient health management data platform, and smart pharmacy in Xixian District. Actively explore new service models such as "basic inspection + hospital diagnosis" and strive to build a regional medical service technology platform to enhance the overall regional medical service capacity.

As the only third-grade public hospital in Xixian New District, our hospital will actively explore the new model of the Xixian. We will strive to make high-quality medical resources flow smoothly, and strive to provide integrated and continuous medical services for disease prevention, diagnosis, treatment, rehabilitation and nursing.

\section{References}

[1] Yu Binbing. The Role, Challenges and Strategies of Large Public Hospitals in Urban Medical Associations [J]. China Management Informationization, 2018(9).

[2] Zhou Hailong. SWOT Analysis of Specialized Medical Association Construction Based on Graded Diagnosis and Treatment [J].Chinese Health Quality Management, 2018(1).

[3] Wang Pei'an. Exploring the Mode of “College Cooperation” Solidly Promoting the Construction of Medical Associations [J]. Jiangsu Health Care Management, 2018 (6).

[4] Wang Yanqun. Discussion on the Role of Medical Association Reform in Promoting Graded Diagnosis and Treatment [J].World Medical Information Digest, 2018(1).

[5] Wang Yajie. "Red Leading” under the Transformation of Medical Association [J]. Chinese Health, 2018(7).

[6]Yan Lihai. Connecting Upper and Down, Smooth Breath Will Not Produce Pain [J]. Chinese Health, 2018 (3). 